\title{
QUANTIFICAÇÃO DE ANTIBIÓTICOS BETA-LACTÂMICOS EM MEDICAMENTOS VETERINÁRIOS: DETERMINAÇÃO DE AMOXICILINA E AMPICILINA POR CROMATOGRAFIA LÍQUIDA DE ALTA EFICIÊNCIA
}

\author{
Gabriela Coelho Miguel, Eduardo Adilson Orlando e Ana Valéria Colnaghi Simionato*,\# \\ Instituto de Química, Universidade Estadual de Campinas, CP 6154, 13083-970 Campinas - SP, Brasil
}

Recebido em 8/11/12; aceito em 30/4/13; publicado na web em 10/6/13

\begin{abstract}
QUANTIFICATION OF BETA-LACTAM ANTIBIOTICS IN VETERINARY DRUGS: AMOXICILLIN AND AMPICILLIN DETERMINATION BY HIGH PERFORMANCE LIQUID CHROMATOGRAPHY. This work focused on the development and validation of an RP-HPLC-UV method for quantification of beta-lactam antibiotics in three pharmaceutical samples. Active principles analyzed were amoxicillin and ampicillin, in 3 veterinary drugs. Mobile phase comprised $5 \mathrm{mmol} \mathrm{L}^{-1}$ phosphoric acid solution at $\mathrm{pH} 2.00$, acetonitrile with gradient elution mode and detection wavelength at $220 \mathrm{~nm}$. The method was validated according to the Brazilian National Health Surveillance regulation, where linear range and linearity, selectivity, precision, accuracy and ruggedness were evaluated. Inter day precision and accuracy for pharmaceutical samples 1,2 and 3 were: 1.43 and $1.43 \% ; 4.71$ and $3.74 \% ; 2.72$ and $1.72 \%$, respectively, while regression coefficients for analytical curves exceeded 0.99 . The method had acceptable merit figure values, indicating reliable quantification. Analyzed samples had active principle concentrations varying from -12 to $+21 \%$ compared to manufacturer label claims, rendering the medicine unsafe for administration to animals.
\end{abstract}

Keywords: beta-lactam antibiotics; HPLC; method validation.

\section{INTRODUÇÃO}

Um medicamento veterinário é definido como toda e qualquer substância que se aplica ou se administra a qualquer animal, para fins terapêuticos, profiláticos ou de diagnóstico, ou mesmo para modificar as funções fisiológicas, de comportamento ou como promotor de crescimento. ${ }^{1}$ Devido à grande aplicação dessa classe de drogas na medicina veterinária e humana, a análise de formulações farmacêuticas, bem como a de fluidos biológicos para o monitoramento de sua biodisponibilidade, perfil farmacocinético e eficiência, é de extrema importância. Além disso, métodos analíticos específicos se fazem necessários para o controle de qualidade das formulações farmacêuticas. ${ }^{2}$

O emprego desses medicamentos para tratamento de animais é permitido e benéfico, mas deve ser utilizado com responsabilidade, já que seu excesso pode provocar a presença de resíduos em tecidos animais destinados à produção de alimentos, ocasionando riscos à saúde humana. Isto ocorre quando o emprego do produto não observa as Boas Práticas de Uso de Medicamentos Veterinários, isto é, o uso oficialmente recomendado ou autorizado, incluindo os períodos de suspensão do tratamento em condições práticas, aprovados por autoridades nacionais. Por isso, a presença de resíduos de medicamentos veterinários em alimentos de origem animal vem sendo monitorada pelos órgãos governamentais responsáveis pela saúde pública, fortalecendo a garantia da segurança alimentar, com consequente diminuição da preocupação dos consumidores. Assim, o monitoramento da concentração do princípio ativo presente na formulação farmacêutica torna-se de suma importância, pois garante que as quantidades adequadas estão sendo administradas e que a finalidade ao qual é destinado será atingida. ${ }^{1}$

Nos últimos anos, o Brasil elevou significativamente seu faturamento no mercado veterinário através da venda de medicamentos como antimicrobianos, antiparasitários, entre outros. Os antimicrobianos, incluindo os antibióticos, tiveram um aumento de 53,8 \% no

*e-mail: avsimionato@iqm.unicamp.br

\#Instituto Nacional de Ciência e Tecnologia em Bioanalítica - INCTBio faturamento em apenas 4 anos. ${ }^{3}$ Por outro lado, o Brasil é o país que mais exporta carne bovina e, atualmente, o mercado de exportação nacional está submetido a uma fiscalização rigorosa, por parte de órgãos internacionais de controle e dos próprios países importadores, quanto à presença de resíduos de medicamentos veterinários nos alimentos de origem animal. ${ }^{4}$ Devido a estes fatos, faz-se necessário o desenvolvimento de métodos analíticos capazes de realizar o monitoramento das formulações farmacêuticas comercializadas.

Os antibióticos são substâncias químicas produzidas sinteticamente, ou através de microorganismos, que destroem (bactericidas) ou inibem o crescimento de outros microorganismos (bacteriostático). ${ }^{3}$ Os antibióticos podem ser diretamente tóxicos ou tornar resistentes os agentes patológicos humanos através de transferência genética, representando um possível risco à saúde humana, além de que, podem produzir reações de hipersensibilidade alérgica. A classe de antibióticos beta-lactâmicos é constituída por uma ampla variedade de moléculas e atualmente tem grande destaque no emprego em medicina veterinária. ${ }^{4}$

Os compostos beta-lactâmicos consistem em uma classe de antibióticos que inclui derivados de penicilinas (Figura 1), cefalosporinas, monobactâmicos, carbapenêmicos e inibidores de beta-lactamases. ${ }^{5}$ Estes antibióticos agem pela inibição da síntese da camada de peptideoglicano, um monômero importante para a integridade da estrutura da parede celular das bactérias, especialmente em organismos Gram-positivos. ${ }^{3}$ Tal processo ocorre pela interação do anel beta-lactâmico do antibiótico com proteínas denominadas PBPs (do inglês, Penicillin Binding Protein), inibindo a enzima envolvida na transpeptidação, responsável pela ligação entre as cadeias de tetrapeptídeos do peptideoglicano. Mais especificamente, os antibióticos beta-lactâmicos inibem a enzima D-alanil-D-alanina transpeptidase, interrompendo a síntese da parede bacteriana. Com isso, há o impedimento da formação das ligações entre os tetrapeptídeos de cadeias adjacentes de peptideoglicano, ocasionando uma perda na rigidez da parede celular. ${ }^{5}$

Nas últimas duas décadas a análise de penicilinas tem sido realizada em diversas amostras, como fármacos, matrizes biológicas e 


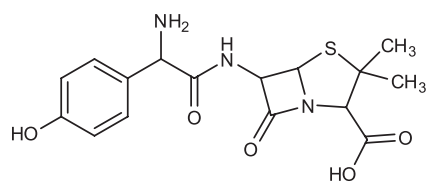

Amoxicilina

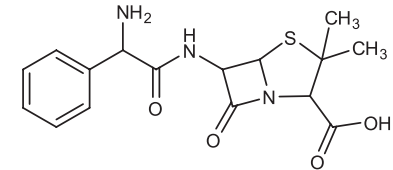

Ampicilina
Figura 1. Estruturas químicas de alguns antibióticos pertencentes à classe das penicilinas, focadas neste trabalho

meios de cultura de fermentação. ${ }^{2}$ Diversas técnicas analíticas têm sido utilizadas nestas análises, tais como: eletroforese capilar - com detecção por espectrometria de massas, fluorescência e absorção de radiação UV-visível; ${ }^{6-11}$ métodos espectrofotométricos; ${ }^{12-14}$ cromatografia líquida de alta eficiência (CLAE) - com detecção por fluorescência, espectrometria de massas e por absorção de radiação UV-visível, ${ }^{15-38}$ sendo a última o método mais utilizado. ${ }^{2}$

Samanidou et al. ${ }^{16}$ demonstraram a possibilidade do uso de cromatografia líquida de alta eficiência em fase reversa com detecção por arranjo de diodos (DAD) para a detecção das penicilinas em formulações farmacêuticas de uso veterinário e em plasma bovino. Foi utilizada uma coluna Perfectsil ODS-2 $(250 \times 4 \mathrm{~mm}, 5 \mu \mathrm{m})$ como fase estacionária, uma solução de ácido trifluoroacético $0,1 \%$ (v/v) como fase móvel aquosa (FM A) e uma mistura ACN:MeOH (90:10 v/v) como fase móvel orgânica (FM B), com eluição no modo gradiente. $\mathrm{O}$ método apresentou boa detectabilidade (LOD variando de 3 a $25 \mathrm{ng}$ ) e exatidão (avaliada por recuperação das penicilinas do plasma bovino com valores de 85,7 a 113,5\%). Cione et al. ${ }^{39}$ também utilizaram CLAE para a determinação de ampicilina em medicamento em pó liofilizado, obtendo exatidão média por recuperação de $99,41 \%$, através de diluição em solução aquosa pH 3,0, separação em coluna X-Terra RP 8 e fase móvel composta portampão fosfato de potássio dibásico pH 3,7 e metanol nas proporções 80:20 (v/v) com eluição no modo isocrático.

Outras técnicas analíticas também são empregadas como descrito por Ma et al. ${ }^{40}$ que utilizaram a capacidade de interação das penicilinas com o complexo Ag(III)-luminol e injeção em fluxo com detecção por quimiluminescência para analisar formulações farmacêuticas, obtendo recuperações entre 103-105 \% para amoxicilina e 100-106 \% para ampicilina. A injeção em fluxo também foi utilizada por Freitas et al. ${ }^{41}$ para determinar amoxicilina em formulações farmacêuticas, com detecção espectrofotométrica do produto formado pela reação de diazotização da o-nitroanilina com amoxicilina em meio alcalino, obtendo limite de detecção de $5,1 \mathrm{mg} \mathrm{L}^{-1}$ e boa precisão, com desvio padrão relativo inferior a 3,9\%. Outra técnica analítica recentemente empregada por Ghoulipour et $a l .^{42}$ para determinar penicilinas em formulações de cápsulas e suspensões foi a cromatografia em camada delgada de alta eficiência, possibilitando limites de quantificação para amoxicilina e ampicilina de 14,5 e 7,5 ng e recuperações de 99,99 e $100,43 \%$, respectivamente.

Neste trabalho usou-se CLAE para separação e quantificação de antibióticos da classe beta-lactâmicos em medicamentos veterinários, a fim de verificar a real concentração destes fármacos na matriz em estudo. Selecionou-se a técnica de CLAE-UV por apresentar sensibilidade adequada para analisar os fármacos na concentração em que se encontram na matriz selecionada, grande aplicabilidade em laboratórios de pesquisa e industriais, além de ser uma técnica bem consolidada, fazendo com que o método desenvolvido e validado possa ser facilmente empregado em demais laboratórios.

\section{PARTE EXPERIMENTAL}

\section{Materiais, regentes e equipamentos}

Os padrões analíticos dos fármacos estudados com pureza de
99\% foram amoxicilina (Fluka Analytical, Alemanha) e ampicilina (Sigma Life Science, Índia). As soluções de trabalho com concentrações de $100 \mu \mathrm{g} \mathrm{mL}^{-1}$ foram preparadas diariamente pela dissolução dos padrões em uma mistura de água:acetonitrila 90:10 (v/v). Para o preparo das fases móveis foram utilizados: acetonitrila, com grau de pureza CLAE, obtida da Tedia (Farfield, USA) e solução aquosa de ácido fosfórico $5 \mathrm{mmol} \mathrm{L} \mathrm{L}^{-1} \mathrm{em} \mathrm{pH}$ 2,00 (Mallinckrodt, Xalostoc, México). Todas as soluções aquosas foram preparadas com água deionizada pelo sistema MilliQ (Millipore - Bedford, MA, USA). As amostras foram dissolvidas em diversos solventes, a fim de determinar o melhor solvente para realizar a extração do princípio ativo da matriz farmacêutica. Os solventes utilizados foram água Milli-Q, ácido fórmico (Carlo Erba, Itália), acetonitrila (Tedia, Farfield, USA), isopropanol (Tedia, Farfield, USA), metanol (Tedia, Farfield, USA) e acetato de etila (LabSynth, Diadema, SP, Brasil).

As amostras de medicamento contendo ampicilina foram preparadas pela simples dissolução do medicamento em água - uma vez que se apresentavam sob a forma de pó. Já as amostras de medicamento contendo amoxicilina, que se apresentavam sob a forma de um veículo oleoso, foram preparadas pela dissolução do medicamento em $50 \mathrm{~mL}$ de ácido fórmico $0,1 \mathrm{~mol} \mathrm{~L}^{-1}$. Este solvente foi empregado na extração da amoxicilina devido ao conhecimento sobre solventes efetivos na extração de fármacos em matrizes farmacêuticas oleosas, obtido em um prévio estudo de solubilidade. As amostras foram centrifugadas por 10 minutos a $60 \mathrm{rpm}$ e então $100 \mu \mathrm{L}$ da fase aquosa foram diluídos em água, resultando em uma concentração final de $25 \mu \mathrm{g} \mathrm{mL}^{-1}$.

No preparo de amostras, empregou-se um Agitador de Soluções AP 52 tipo vórtex da Phoenix Luferco e uma centrífuga Rotofix 32A da Hettich Zentrifugen, para melhor homogeneização das soluções.

\section{Análise por CLAE}

Para as análises cromatográficas, utilizou-se um cromatógrafo a líquido modelo Shimadzu Proeminence, com detector por absorção de radiação ultravioleta com arranjo de diodos (UV DAD) Shimadzu SPD-M20A - Photodiode array detector, com bombas reciprocantes LC-20AT. Usou-se uma coluna com fase estacionária octadecilsilano, modelo ACE C18 (Advanced Chromatography Technologies, Aberdeen, UK), com as dimensões $150 \mathrm{~mm} \times 4,6 \mathrm{~mm}$ e tamanho de partícula de $3 \mu \mathrm{m}$.

Após desenvolvimento do método de análise foram selecionadas as condições ótimas para a separação cromatográfica dos 2 antibióticos, como: fase móvel aquosa composta por solução de ácido fosfórico 5 mmol L-1 pH 2,00 (FM A); fase móvel orgânica: acetonitrila (FM B); vazão da FM: $1 \mathrm{~mL} \mathrm{~min}^{-1}$; modo de eluição em gradiente (em \% FM B): 0 min - 0\%; $6 \min -30 \% ; 11 \min -70 \%$; $18 \min -0 \% ; 25$ min - 0\%; comprimento de onda de detecção: $220 \mathrm{~nm}$; coluna ACE (fase estacionária C18).

Para o preparo da fase móvel otimizada, preparou-se $900 \mathrm{~mL}$ de uma solução de ácido fosfórico, através da diluição de $300 \mu \mathrm{L}$ de ácido fosfórico concentrado em $900 \mathrm{~mL}$ de água, sendo esta a fase móvel aquosa. $\mathrm{O}$ pH da solução foi ajustado para 2,00 com ácido fosfórico concentrado, com auxílio de um pHmetro. A fase móvel orgânica foi apenas filtrada em um sistema de filtração a vácuo com filtro de membrana de politetrafluoretileno com poros de $0,45 \mu \mathrm{m}$ (Sartorius, Alemanha), procedimento que também foi realizado com a solução aquosa, porém com membrana de celulose com poros de $0,45 \mu \mathrm{m}$ (Schleicher \& Schuell, Brasil). Ambas as soluções foram estocadas em frascos de vidro e armazenadas à temperatura ambiente, ao abrigo de luz. 


\section{Validação do método analítico}

A validação do método analítico constitui o primeiro nível de garantia da qualidade em um laboratório. Um método é considerado validado se atende às especificações da legislação vigente. Para técnicas analíticas cromatográficas em fármacos e matrizes biológicas, a validação é regulamentada no Brasil pela Agência Nacional de Vigilância Sanitária (ANVISA), segundo a Resolução $n^{\circ} 899$, de 29 de maio de 2003, e exige que os seguintes parâmetros, ou figuras de mérito, sejam satisfeitos na análise de fármacos: especificidade (seletividade), faixa linear, linearidade, precisão e robustez. ${ }^{1,43-45}$ Esse processo de validação do método analítico é fundamental para confiabilidade do resultado obtido e tem primordial interesse no ramo farmacêutico devido ao grande impacto de uma possível falha de produção. ${ }^{46} \mathrm{O}$ método foi validado com o uso de medicamentos contendo os princípios ativos de interesse, fazendo com que as figuras de mérito obtidas fossem mais fidedignas. Tais princípios são comumente encontrados sob a forma de suspensão injetável ou em pó solúvel.

\section{Linearidade}

A linearidade demonstra que o método fornece resultados proporcionais à concentração do analito na amostra, dentro da faixa linear da curva analítica de, no mínimo, 5 concentrações diferentes, expressada pelo coeficiente de correlação (r), que deve ser maior que 0,99 . Já a faixa linear é a faixa de concentração na qual a sensibilidade se mantém constante, sendo derivada normalmente do estudo da linearidade e depende da aplicação pretendida. Em um ensaio para determinação quantitativa do analito em matérias-primas ou em formulações farmacêuticas, o alcance deve ser de $80 \%$ a $120 \%$ da concentração teórica do teste. ${ }^{1,43}$

A linearidade do método foi avaliada pela construção de curvas analíticas com soluções de padrão em solvente em diferentes concentrações, em triplicata, através do método do padrão externo.

\section{Seletividade}

A fim de determinar se o método desenvolvido era seletivo, submeteram-se os compostos analisados a condições de estresse, tais como dissolução dos padrões em solução ácida, básica, oxidante e em solução aquosa sob aquecimento. O objetivo deste procedimento era provocar a degradação dos padrões e, assim, determinar se algum produto de degradação poderia coeluir com algum composto durante a análise cromatográfica. O método é considerado seletivo nas condições em que não há coeluição entre os analitos e qualquer produto de degradação formado, sendo então apenas necessária a comparação entre os perfis cromatográficos das amostras analisadas para avaliação da seletividade.

As análises foram realizadas em intervalos de $1 \mathrm{~h}$ e 24 h após o preparo das soluções e os cromatogramas, obtidos para as análises em cada intervalo de tempo, foram comparados para verificar possíveis coeluições entre os analitos.

\section{Precisão intra e inter-dias}

A precisão envolve 3 níveis: repetibilidade, ou seja, resultados semelhantes efetuados por um mesmo analista em um mesmo instrumento dentro de um curto período de tempo; precisão intermediária, que é a concordância entre resultados obtidos em dias diferentes; e reprodutibilidade, que avalia resultados de diferentes laboratórios. ${ }^{1,43,47}$ De acordo com a ANVISA, o coeficiente de variação deve ser menor que $5 \%$ para que o método seja considerado preciso, enquanto que, de acordo com a Farmacopéia Britânica, ${ }^{48}$ deve ser menor que $1 \%$.

As soluções empregadas na determinação da precisão do método foram obtidas através do preparo de uma solução-estoque contendo o medicamento, para posterior diluição, gerando a solução de trabalho. No caso da ampicilina, dissolveu-se $25,0 \mathrm{mg}$ de princípio ativo contido no medicamento em $100 \mathrm{~mL}$ de água deionizada. As soluções de trabalho foram preparadas através da diluição da solução-estoque, de modo que se obtivesse uma concentração igual à metade da faixa linear determinada pela curva analítica $\left(25 \mu \mathrm{g} \mathrm{mL}^{-1}\right)$. Já para a validação do método para quantificação da amoxicilina, preparou-se a solução-estoque dissolvendo-se $0,833 \mathrm{~mL}$ do medicamento em 50 $\mathrm{mL}$ de ácido fórmico $0,1 \mathrm{~mol} \mathrm{~L}^{-1}$, seguido de centrifugação por 10 minutos a uma velocidade de rotação de $60 \mathrm{rpm}$ e, então, diluição de uma alíquota de $100 \mu \mathrm{L}$ em $10 \mathrm{~mL}$ de água, produzindo-se uma solução de trabalho de $25 \mu \mathrm{g} \mathrm{mL} \mathrm{m}^{-1}$.

\section{Exatidão}

A exatidão resulta tanto de erros aleatórios quanto sistemáticos, porém, sendo o primeiro dificilmente mensurável, a exatidão é diretamente relacionada a erros sistemáticos, que podem ser identificados e eliminados. Para avaliar a exatidão de um método analítico podem ser usados materiais de referência, realizar comparação entre métodos, fazer ensaios de recuperação ou construir curvas analíticas pelo método da adição-padrão. ${ }^{43,47}$

Após a avaliação da precisão do método, foi realizada a análise da exatidão do mesmo. Inicialmente, optou-se pelo método do padrão externo, devido ao menor tempo requerido para execução do mesmo. Assim, prepararam-se em triplicata soluções contendo $25 \mu \mathrm{g} \mathrm{mL} \mathrm{m}^{-1}$ de ampicilina, sendo que destes, $36 \%$ eram oriundos do princípio ativo presente no medicamento e $64 \%$ do padrão analítico e vice-versa.

A porcentagem de analito recuperada foi calculada através da relação entre a concentração experimental encontrada na análise e a concentração teórica obtida a partir da fortificação com padrão, em termos de porcentagem (Equação 1).

$$
\text { Exatidão }=\mathrm{R}=\frac{\text { concentração média experimental }}{\text { concentração teórica }} \times 100
$$

Entretanto, devido à presença de efeito matriz (dados não apresentados), obtiveram-se valores de recuperação fora da faixa considerada válida (entre $95 \%$ e $105 \%$ da concentração). Deste modo, foi necessária a quantificação da ampicilina na formulação através da técnica da adição de padrão.

Assim, prepararam-se, em triplicata, cinco soluções contendo o analito proveniente do medicamento em questão na concentração de $20 \mu \mathrm{g} \mathrm{mL}^{-1}$ (o que equivale a $80 \%$ do valor do ponto médio da curva) e o padrão nas concentrações de $0,10,20,30$ e $40 \%$ com relação ao mesmo ponto, a fim de que todas as concentrações estivessem contidas na faixa linear da curva de trabalho - entre 20 e $30 \mu \mathrm{g} \mathrm{mL}^{-1}$. As adições de padrão foram feitas a partir de uma solução estoque de ampicilina de concentração $100 \mu \mathrm{g} \mathrm{mL}^{-1}$. A exatidão foi expressa através do $\mathrm{CV}$ entre as concentrações obtidas na determinação dos três gráficos, devendo ser igual ou inferior a $5 \%$.

Como havia grande possibilidade do medicamento contendo a amoxicilina também apresentar efeito matriz, devido às semelhanças estruturais dos dois compostos, optou-se por realizar a avaliação da exatidão deste analito diretamente pelo método da adição de padrão, seguindo o mesmo procedimento citado anteriormente para a ampicilina, porém com uma solução estoque de amoxicilina na concentração de $2000 \mu \mathrm{g} \mathrm{mL} \mathrm{m}^{-1}$. 


\section{Robustez}

Para avaliação da robustez foram realizadas pequenas variações em alguns parâmetros do método e consequente influência nos resultados. Mais especificamente, em CLAE, essas variações podem ser oriundas de mudanças de $\mathrm{pH}$, composição, natureza e fluxo da fase móvel, temperatura da coluna entre outros. A avaliação destas figuras de mérito são necessárias para o analista ter conhecimento sobre quais parâmetros de análise devem ser cuidadosamente controlados, a fim de evitar variações nos resultados. ${ }^{43}$

A robustez do método foi avaliada segundo o teste de Youden. ${ }^{47}$ Trata-se de um teste que permite não só avaliar a robustez do método, como também ordenar e classificar a influência de cada uma das variações nos resultados finais.

Foram selecionados sete fatores ou condições para as quais a introdução de pequenas variações poderia afetar os resultados da etapa de preparo de amostra e do método cromatográfico, mas sem descaracterizar os princípios gerais de extração dos analitos, separação cromatográfica ou detecção. (Tabela 1). Posteriormente, oito ensaios foram estabelecidos através da combinação dos sete fatores selecionados (Tabela 2), seguindo as orientações do INMETRO. ${ }^{49}$

O preparo das amostras foi realizado pesando-se o medicamento em balança analítica de forma a obter $25,0 \mathrm{mg}$ de princípio ativo e então dissolvendo-os em $100 \mathrm{~mL}$ de água deionizada ou em $100 \mathrm{~mL}$ de uma mistura de $\mathrm{H}_{2} \mathrm{O}: \mathrm{ACN}(95: 5 \mathrm{v} / \mathrm{v})$. Cada amostra foi dividida em duas partes e uma delas permaneceu em ultrassom por 15 minutos. As soluções de trabalho foram preparadas através da diluição da solução-estoque, para obtenção de solução com concentração igual à metade da faixa linear determinada pela curva analítica $\left(25 \mu \mathrm{g} \mathrm{mL} \mathrm{m}^{-1}\right)$. Tais amostras foram preparadas e analisadas em duplicata e em ordem aleatória.

As respostas avaliadas pela matriz elaborada para cada um dos ensaios foram as áreas dos picos cromatográficos (Tabela 2 - s, t, u, v, w, x, y e z). Observando-se as linhas da Tabela 2, o efeito da variação de cada fator é estimado encontrando-se os quatro resultados correspondentes aos valores positivos e aos quatro resultados negativos e fazendo-se a diferença entre as médias dos dois grupos.

Tabela 1. Parâmetros selecionados para o teste de Youden, com seus valores nominais e suas respectivas variações

\begin{tabular}{|c|c|c|c|}
\hline Fator & Parâmetros & $\begin{array}{c}\text { Condição } \\
\text { nominal }(+1)\end{array}$ & Variação (-1) \\
\hline $\mathbf{A}$ & $\begin{array}{l}\text { Proporção inicial da FM } \\
\text { (ACN: solução de } \mathrm{H}_{3} \mathrm{PO}_{4} \text { ) }\end{array}$ & $0: 100$ & 2:98 \\
\hline B & $\mathrm{pH}$ da FM aquosa & 2,00 & 2,20 \\
\hline $\mathbf{C}$ & $\begin{array}{l}\text { Ultrassom para o preparo } \\
\text { da amostra }\end{array}$ & - & $15 \mathrm{~min}$ \\
\hline D & $\begin{array}{l}\text { Solubilização da amostra } \\
\left(\mathrm{ACN}: \mathrm{H}_{2} \mathrm{O}\right)\end{array}$ & 0:100 & $5: 95$ \\
\hline $\mathbf{E}$ & $\begin{array}{c}\text { Comprimento de onda de } \\
\text { detecção }(\lambda)\end{array}$ & $220 \mathrm{~nm}$ & $221 \mathrm{~nm}$ \\
\hline $\mathbf{F}$ & Vazão da fase móvel & $1,0 \mathrm{~mL} / \mathrm{min}$ & $1,1 \mathrm{~mL} / \mathrm{min}$ \\
\hline G & $\begin{array}{l}\text { Fabricante da Fase Orgânica } \\
\qquad(\mathrm{ACN})\end{array}$ & Tedia & J. T. Baker \\
\hline
\end{tabular}

Os valores dos efeitos (E) de cada um dos $\mathrm{N}$ fatores foram calculados de acordo com a Equação $2 .^{47}$

$$
\mathrm{E}_{\mathrm{N}}=\frac{\sum \mathrm{Y}(+1)-\sum \mathrm{Y}(-1)}{4}
$$

onde $\Sigma \mathrm{Y}(+1)$ e $\Sigma \mathrm{Y}(-1)$ representam a soma das respostas dos níveis $(+1)$ e $(-1)$, respectivamente, e o denominador provém de N/2.
Tabela 2. Matriz referente a combinação dos fatores avaliados para a determinação da robustez do método

\begin{tabular}{ccccccccc}
\hline \multirow{2}{*}{ Fator } & \multicolumn{7}{c}{$\mathrm{N}^{\circ}$ do Ensaio } \\
\cline { 2 - 8 } & $\mathbf{1}$ & $\mathbf{2}$ & $\mathbf{3}$ & $\mathbf{4}$ & $\mathbf{5}$ & $\mathbf{6}$ & $\mathbf{7}$ & $\mathbf{8}$ \\
\hline $\mathbf{A}$ & +1 & +1 & +1 & +1 & -1 & -1 & -1 & -1 \\
$\mathbf{B}$ & +1 & +1 & -1 & -1 & +1 & +1 & -1 & -1 \\
$\mathbf{C}$ & +1 & -1 & +1 & -1 & +1 & -1 & +1 & -1 \\
$\mathbf{D}$ & +1 & +1 & -1 & -1 & -1 & -1 & +1 & +1 \\
$\mathbf{E}$ & +1 & -1 & +1 & -1 & -1 & +1 & -1 & +1 \\
$\mathbf{F}$ & +1 & -1 & -1 & +1 & +1 & -1 & -1 & +1 \\
$\mathbf{G}$ & +1 & -1 & -1 & +1 & -1 & +1 & +1 & -1 \\
\hline Resposta & $\mathbf{s}$ & $\mathbf{t}$ & $\mathbf{u}$ & $\mathbf{v}$ & $\mathbf{w}$ & $\mathbf{x}$ & $\mathbf{y}$ & $\mathbf{z}$ \\
(área do pico) & & & & & & & & \\
\hline
\end{tabular}

(+): uso da condição nominal; (-): uso da variação (Tabela 1).

Os efeitos foram então normalizados com relação à área média nominal do(s) pico(s), isto é, aquelas onde foram empregadas somente as condições nominais (Equação 3).

$$
\% \mathrm{E}_{\mathrm{N}}=\frac{\mathrm{E}_{\mathrm{N}}}{\overline{\mathrm{Y}}} \times 100 \%
$$

onde $\overline{\mathrm{Y}}$ é a média dos resultados nominais.

$\mathrm{O}$ método é considerado robusto para determinado fator se $-5 \%<\% \mathrm{E}_{\mathrm{N}}<+5 \%$.

\section{RESULTADOS E DISCUSSÕES}

Inicialmente o método de análise para separação da amoxicilina (AMO) e ampicilina (AMP) foi desenvolvido pela avaliação de diversas condições cromatográficas, tais como: natureza da fase móvel orgânica (metanol e acetonitrila), composição (ácido acético, ácido fórmico e ácido fosfórico) e pH da fase móvel aquosa selecionada (ácido fosfórico, nos pHs 2,0 a 4,5), gradiente de fase móvel, vazão da fase móvel, fases estacionárias (fenil e octadecilsilano) e dimensões da coluna cromatográfica e respectivos tamanhos de partículas de fase estacionária (dados não apresentados). As condições ótimas de separação foram obtidas através da avaliação de parâmetros cromatográficos, como resolução entre pares de picos (mínimo de 1,5) e eficiência, bem como tempo de análise (Figura 2). Assim, usando tais condições, foram obtidos valores de resolução de 4,11 para os pares de picos da AMO e AMP.

A validação demonstra que o método é adequado ao uso pretendido e é fundamental quando decisões importantes são baseadas nos resultados obtidos pelo mesmo. A validação é uma boa oportunidade de identificar os pontos fortes e fracos do método e, uma vez concluída, considera-se o mesmo consolidado e adequado ao seu propósito, gerando resultados confiáveis e podendo ser reproduzido em outros laboratórios com garantia da qualidade dos dados gerados. Devem ser considerados vários fatores quando a validação é feita, como máximo nível de resíduo, toxicidade, grau de automação do método, custos, tamanho da amostra, analitos e matrizes.

\section{Validação do método para análise de medicamentos contendo ampicilina}

Iniciou-se a validação do método para quantificação do fármaco ampicilina, usando uma formulação injetável, aqui denominada de medicamento 1, o qual é apresentado em ampola de vidro com $2 \mathrm{~g}$ de ampicilina sódica em pó e uma ampola com $10 \mathrm{~mL}$ de água destilada, 
Tabela 3. Avaliação da repetibilidade e precisão intermediária do método cromatográfico para a análise de ampicilina no medicamento 1

\begin{tabular}{|c|c|c|c|c|c|}
\hline \multicolumn{3}{|c|}{ Repetibilidade } & \multicolumn{3}{|c|}{ Precisão intermediária } \\
\hline $\begin{array}{c}\text { Concentração nominal } \\
\left(\mu \mathrm{g} \mathrm{mL} L^{-1}\right)\end{array}$ & $\begin{array}{c}\text { Concentração experimen- } \\
\left.\text { tal }(\mu \mathrm{g} \mathrm{mL})^{-1}\right)^{*}\end{array}$ & $\mathrm{CV}(\%)$ & $\begin{array}{c}\text { Concentração nominal } \\
\left(\mu \mathrm{g} \mathrm{mL} L^{-1}\right)\end{array}$ & $\begin{array}{c}\text { Concentração experimen- } \\
\left.\operatorname{tal}(\mu \mathrm{g} \mathrm{mL})^{-1}\right)^{* *}\end{array}$ & $\mathrm{CV}(\%)$ \\
\hline 25 & $21,86 \pm 0,22$ & 0,99 & 25 & $21,65 \pm 0,31$ & 1,43 \\
\hline Curvas analíticas $* * *$ & $\begin{array}{c}y=23454 x+8072 \\
R^{2}=0,99566\end{array}$ & \multicolumn{2}{|c|}{$\begin{array}{c}y=21993,8 x+17050,8 \\
R^{2}=0,99374\end{array}$} & \multicolumn{2}{|c|}{$\begin{array}{c}y=22182,1 x+16897 ; \\
R^{2}=0,99616\end{array}$} \\
\hline
\end{tabular}

$* \mathrm{n}=6 . * * \mathrm{n}=12$. ***As curvas analíticas são representadas pela equação da reta: $y=a x+b$, onde: $y$ é a área do pico cromatográfico; $x$ é a concentração do analito; $a$ é a inclinação da reta; $b$ é o intercepto da reta com o eixo $y ; R^{2}$ : é o coeficiente de regressão linear da curva.

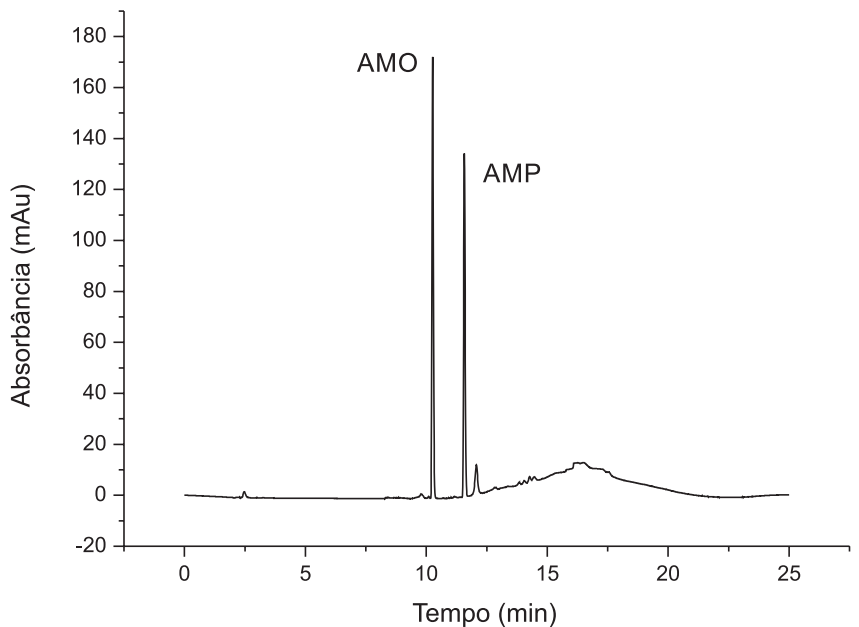

Figura 2. Separação dos antibióticos beta-lactâmicos amoxicilina (AMO) e ampicilina $(A M P)$ por CLAE. As soluções analisadas foram preparadas através da dissolução de padrão analítico em solvente, resultando em soluções de $25 \mu \mathrm{g} \mathrm{mL} L^{-1}$. Todas as demais condições cromatográficas estão descritas na parte experimental deste estudo

para dissolução. Este medicamento é indicado para tratamento de infecções respiratórias de aves, gatos e coelhos, infecções gastrointestinais, infecções urinárias, entre outras, nos quais os antígenos sejam sensíveis à ampicilina. Avaliou-se primeiramente a precisão do método, seguindo as especificações da ANVISA para métodos analíticos. ${ }^{1}$ Desta forma, a precisão foi determinada em dois níveis: repetibilidade e precisão intermediária.

Avaliou-se a repetibilidade do método através da variação das áreas dos picos cromatográficos observados na análise de seis amostras com mesma concentração. Após construção da curva analítica para o fármaco ampicilina pelo método do padrão externo, a precisão foi determinada pela análise de uma solução do medicamento 1 - em replicata e no mesmo dia - contendo $25 \mu \mathrm{g} \mathrm{mL}^{-1}$ do fármaco. A partir das áreas dos picos cromatográficos e da equação da reta da curva analítica, calculou-se a concentração do analito em cada análise e então foi obtido o coeficiente de variação (CV) do método, que demonstra se o mesmo apresenta repetibilidade aceitável (Tabela 3). De acordo com a ANVISA, o CV deve ser menor que 5\%, enquanto que, de acordo com a Farmacopéia Britânica, ${ }^{48}$ deve ser menor que $1 \%$. Desta forma, considera-se que o método apresenta precisão intra dia adequada, já que foi obtido um CV de 0,99\% para as 6 análises realizadas.

Da mesma forma, foram feitas análises cromatográficas de amostras recém-preparadas em dois dias diferentes e não consecutivos para determinar a precisão interdia do método. Para cada dia foi construída uma curva analítica pelo método do padrão externo a fim de tornar os dados de precisão mais confiáveis, pois, dessa forma, a determinação da concentração do analito fica menos suscetível à erros sistemáticos e aleatórios. A cada dia de análise as amostras foram preparadas da mesma forma que na análise de repetibilidade, porém as análises foram feitas em triplicata. No final do terceiro dia, agrupou-se os valores de concentração do fármaco calculados nos 3 dias de análise e calculou-se o CV da média das concentrações (Tabela 3). Como o CV obtido foi inferior a 5\%, considera-se que o método também é preciso em nível intermediário e, então, averiguou-se a exatidão do mesmo. ${ }^{1}$

A exatidão do método foi avaliada através de testes de recuperação. Assim, expressou-se a exatidão através do coeficiente de variação entre as recuperações obtidas nas replicatas dos dois conjuntos de amostras, seguindo um critério de aceitação: $95 \%<\mathrm{R}<105 \%$ e $\mathrm{CV}<5 \%$, segundo a Equação 1.

Construíram-se gráficos relacionando as concentrações dos padrões e as respectivas áreas obtidas. O ponto onde a reta corta o eixo y corresponde à área do pico do analito na amostra, sem qualquer adição do padrão. ${ }^{7}$ A extrapolação da reta define, no eixo x, a concentração do analito na amostra. A exatidão foi expressa através do CV entre as concentrações obtidas na determinação dos três gráficos, devendo ser igual ou inferior a $5 \%$. Os valores de recuperação encontrados estão contidos na Tabela 4

Tabela 4. Concentrações e recuperações obtidos para as soluções com maior e menor porcentagem de ampicilina proveniente do medicamento 1 , respectivamente, através do método do padrão externo

\begin{tabular}{ccc}
\hline $\mathrm{C}_{\text {Med }} / \mu \mathrm{g} \mathrm{mL}-1$ & $\mathrm{C}_{\mathrm{Pad}} / \mu \mathrm{g} \mathrm{mL} \mathrm{Lec}^{-1}$ & 120,68 \\
\hline 13,99 & 10,9 & 112,76 \\
& 10,1 & 116,91 \\
\hline & 10,5 & 116,78 \\
Média & 10,5 & 3,96 \\
$\mathrm{SD}$ & 0,36 & 3,39 \\
$\mathrm{CV}$ & - & 117,87 \\
\hline 7,87 & 18,9 & 131,56 \\
& 21,1 & 130,98 \\
\hline Média & 21,0 & 126,81 \\
$\mathrm{SD}$ & 20,3 & 7,74 \\
$\mathrm{CV}$ & 1,24 & 6,10 \\
\hline
\end{tabular}

$\mathrm{C}_{\mathrm{Med}}=$ Concentração de ampicilina proveniente do medicamento; $\mathrm{C}_{\mathrm{Pad}}=$ Concentração de ampicilina proveniente do padrão analítico; \% Rec $=$ Porcentagem de recuperação; $\mathrm{SD}=$ Desvio padrão; $\mathrm{CV}=$ Coeficiente de variação

Neste primeiro experimento, as recuperações obtidas ultrapassaram o limite máximo permitido (105\%) e, portanto, o medicamento apresenta efeito matriz e sua quantificação somente pode ser efetuada pelo método de adição de padrão.

Para a adição de padrão, foram preparadas amostras de medicamentos em triplicatas contendo 0 a $8 \mu \mathrm{g} \mathrm{mL} \mathrm{m}^{-1}$ do padrão de 
Tabela 5. Avaliação da exatidão do método cromatográfico para a análise de ampicilina no medicamento 1

\begin{tabular}{|c|c|c|c|c|c|}
\hline & Curvas analíticas* & & $\begin{array}{c}\text { Concentração nominal } \\
\left(\mu \mathrm{g} \mathrm{mL} L^{-1}\right)\end{array}$ & $\begin{array}{c}\text { Concentração experimental } \\
\left(\mu \mathrm{g} \mathrm{mL} \mathrm{L}^{-1}\right)^{* *}\end{array}$ & $\mathrm{CV}(\%)$ \\
\hline $\begin{array}{c}y=23145 x+411578 \\
R^{2}=0,9979\end{array}$ & $\begin{array}{c}y=23643 x+409065 \\
R^{2}=0,9965\end{array}$ & $\begin{array}{c}y=23119 x+408562 \\
R^{2}=0,9966\end{array}$ & 20 & $17,58 \pm 0,25$ & 1,43 \\
\hline
\end{tabular}

*As curvas analíticas são representadas pela equação da reta: $y=a x+b$, onde: $y$ é a área do pico cromatográfico; $x$ é a concentração do analito; $a$ é a inclinação da reta; $b$ é o intercepto da reta com o eixo $y ; R^{2}$. é o coeficiente de regressão linear da curva. $* * n=3$.

ampicilina, com incrementos de $2 \mu \mathrm{g} \mathrm{mL} \mathrm{m}^{-1}$. Assim, a concentração do fármaco na amostra original foi determinada pela extrapolação da curva analítica ao eixo $x$ e o CV foi calculado a partir da média das três concentrações obtidas. Concomitantemente, o valor médio das concentrações experimentais foi comparado com a concentração teórica para cálculo da recuperação, observando-se $\mathrm{CV} \leq 5 \%$, o que define o método como exato (Tabela 5). A concentração de princípio ativo encontrada no medicamento está cerca de $12,6 \%$ abaixo do valor nominal de $20 \mu \mathrm{g} \mathrm{mL}^{-1}$ - definido pelo preparo da amostra. Como a bula do medicamento indica que o mesmo apresenta concentração total do fármaco de $200.000 \mu \mathrm{g} \mathrm{mL} L^{-1}$, a concentração real é de aproximadamente 175.854,9 $\mu \mathrm{g} \mathrm{mL}^{-1}$. Este dado corrobora com o valor obtido anteriormente na etapa de precisão, sendo aproximadamente $12,1 \%$ abaixo do valor esperado.

A avaliação da robustez não é sempre realizada em processos de validação devido a sua complexidade e falta de protocolos bem estabelecidos. Embora tal avaliação ainda não seja obrigatória na validação de um método, alguns órgãos (como o FDA - the US Food and Drug Administration $)^{50}$ a exigem para o registro de novos medicamentos. ${ }^{47}$ Assim, aplicou-se o teste de Youden para avaliar a robustez do método analítico para a determinação de ampicilina no medicamento $1 .{ }^{47} \mathrm{O}$ método é considerado robusto para determinado fator se $-5 \%<\% \mathrm{E}_{\mathrm{N}}<+5 \%$ (Equação 3). A Tabela 6 mostra que o método é robusto para cinco dos sete parâmetros avaliados. Desta forma, pequenas alterações: a) na proporção inicial da fase móvel orgânica em relação à fase móvel aquosa; $b$ ) no $\mathrm{pH}$ da fase móvel aquosa; c) no solvente usado para solubilização da amostra; d) no fabricante da fase móvel orgânica; e) no uso de ultrassom para o preparo da amostra, não influenciam consideravelmente os resultados obtidos nas análises. No entanto, pequenas alterações no comprimento de onda de detecção e na vazão da fase móvel apresentam uma maior influência na qualidade dos resultados obtidos, sendo então necessário controlar mais rigorosamente tais parâmetros. Como o modo de eluição cromatográfica é feito por gradiente, o controle da vazão da fase móvel é crítico para que a retenção da ampicilina não seja apreciavelmente alterada e a linha de base permaneça estável ao longo de toda análise. Já a alteração do comprimento de onda foi responsável por uma grande variação na área do pico, entretanto, aliado à variação de outros parâmetros, o valor final de $\mathrm{E}_{\mathrm{N}}$ ultrapassou apenas ligeiramente o limite estabelecido. É importante ressaltar que o comprimento de onda monitorado $(220 \mathrm{~nm})$ representa um compromisso entre maior intensidade do pico cromatográfico - culminando em melhor quantificação da ampicilina - e seletividade (por menor detecção de interferentes).

Após validação do método para quantificação do princípio ativo ampicilina no medicamento 1 , realizou-se nova validação para
Tabela 6. Valores dos efeitos calculados para cada um dos parâmetros investigados no teste de Youden, na avaliação da robustez do método cromatográfico para a análise de ampicilina no medicamento 1

\begin{tabular}{ll}
\hline Parâmetro & Efeito $(\%)$ \\
\hline Proporção inicial da FM $\left(\mathrm{ACN}: \mathrm{H}_{3} \mathrm{PO}_{4}\right)$ & $-2,363$ \\
\hline pH da FM aquosa & 0,413 \\
\hline Ultrassom para o preparo da amostra & 0,144 \\
\hline Solubilização da amostra $\left(\mathrm{ACN}: \mathrm{H}_{2} \mathrm{O}\right)$ & 1,485 \\
\hline Comprimento de onda $(\lambda)$ & 6,136 \\
\hline Vazão & 8,127 \\
\hline Marca da Fase Móvel Orgânica $(\mathrm{ACN})$ & 0,740 \\
\hline
\end{tabular}

quantificação do mesmo princípio ativo em um medicamento de administração oral, aqui denominado medicamento 2. Sua apresentação é semelhante ao anterior, em um frasco de vidro contendo $50 \mathrm{~g}$ de pó para reconstituição em água e trata-se de um antibiótico que atua contra infecções em cães, gatos e aves, causadas por bactérias Gramnegativas sensíveis à ampicilina. O procedimento de validação foi o mesmo apresentado para o medicamento 1 e os resultados obtidos são apresentados na Tabela 7.

A exatidão foi avaliada pela construção de curvas analíticas pelo método da adição de padrão, pois assim poder-se-ia determinar diretamente a quantidade de princípio ativo presente nesta formulação, além de eliminar a possibilidade da influência de efeito matriz. A exatidão foi expressa através do CV entre as concentrações obtidas pelas três curvas elaboradas, devendo ser igual ou inferior a $5 \%$.

A validação feita para o medicamento 2 mostrou que o método é preciso (nos dois níveis avaliados) e exato, de acordo com as normas estabelecidas pela ANVISA. ${ }^{1}$ A robustez do método não foi investigada para o medicamento 2, pois essa figura de mérito já havia sido averiguada para o medicamento 1, considerando-se então que o método é robusto para cinco das sete variáveis avaliadas. A concentração de princípio ativo encontrada no medicamento 2 está cerca de 4,16\% acima do valor indicado pelo fabricante. Assim, como a concentração de ampicilina no medicamento era de $200.000 \mu \mathrm{g} \mathrm{mL}{ }^{-1}$, a concentração real de princípio ativo na formulação é de $208.312,6 \mu \mathrm{g} \mathrm{mL}^{-1}$.

\section{Validação do método para análise de medicamentos contendo amoxicilina}

A validação do método para quantificação de amoxicilina foi feita pela análise do medicamento 3, o qual é apresentado como uma ampola de vidro contendo $100 \mathrm{~mL}$, sendo $15 \mathrm{~g}$ de amoxicilina

Tabela 7. Avaliação da precisão e exatidão do método cromatográfico para a análise de ampicilina no medicamento 2

\begin{tabular}{cccccccccc}
\hline & Repetibilidade & \multicolumn{4}{c}{ Precisão intermediária } & \multicolumn{3}{c}{ Exatidão } \\
\hline $\mathrm{C}_{\mathrm{med}}(\mu \mathrm{g} \mathrm{mL})^{*}$ & $\mathrm{SD}$ & $\mathrm{CV}(\%)$ & $\left.\mathrm{C}_{\mathrm{med}}(\mu \mathrm{g} \mathrm{mL})^{-1}\right)^{* *}$ & $\mathrm{SD}$ & $\mathrm{CV}(\%)$ & $\mathrm{C}_{\mathrm{med}}(\mu \mathrm{g} \mathrm{mL})^{* * *}$ & $\mathrm{SD}$ & $\mathrm{CV}(\%)$ \\
\hline 21,14 & 0,20 & 0,95 & 21,14 & 1,00 & 4,71 & 20,83 & 0,74 & 3,54 & \\
\hline
\end{tabular}

$\mathrm{C}_{\text {med }}=$ concentração média do fármaco no medicamento. $* \mathrm{n}=3 . * * \mathrm{n}=12 . * * * \mathrm{n}=3$. 
Tabela 8. Avaliação da precisão e exatidão do método cromatográfico para a análise de amoxicilina no medicamento 3

\begin{tabular}{ccccccccc}
\hline & \multicolumn{4}{c}{ Precisão intermediária } & \multicolumn{3}{c}{ Exatidão } \\
\hline $\mathrm{C}_{\text {med }}(\mu \mathrm{g} \mathrm{mL})^{-1} *$ & $\mathrm{SD}$ & $\mathrm{CV}(\%)$ & $\mathrm{C}_{\text {med }}(\mu \mathrm{g} \mathrm{mL})^{-1} * *$ & $\mathrm{SD}$ & $\mathrm{CV}(\%)$ & $\mathrm{C}_{\text {med }}(\mu \mathrm{g} \mathrm{mL})^{-1} * * *$ & $\mathrm{SD}$ & $\mathrm{CV}^{*}(\%)$ \\
\hline 22,37 & 0,42 & 1,70 & 24,38 & 0,66 & 2,72 & 24,25 & 0,42 & 1,72 \\
\hline$* \mathrm{n}=3 . * * \mathrm{n}=12 . * * * \mathrm{n}=3$. & & & & &
\end{tabular}

triidratada e $100 \mathrm{~mL}$ de veículo. Trata-se de uma solução injetável e apresentada na forma de veículo oleoso, usado no controle ou tratamento de infecções por microrganismos sensíveis à amoxicilina em bovinos, ovinos, caprinos, suínos, cães e gatos. Após estudo do melhor método para extração do princípio ativo da matriz oleosa, concluiu-se que a solubilização do medicamento em ácido fórmico $0,1 \mathrm{~mol} \mathrm{~L}^{-1}$ seguido por diluição em água era o procedimento mais adequado, já que a amoxicilina é altamente solúvel em soluções ácidas aquosas (dados não apresentados).

A precisão do método de análise foi estudada pela construção de curvas analíticas usando o método do padrão externo, tal como citado no item 3.1. Já a exatidão foi avaliada pela construção de curvas analíticas pelo método da adição de padrão, que leva à determinação direta da concentração do analito na amostra, podendo então calcular o coeficiente de variação em relação à concentração nominal (Tabela 8). Como os coeficientes de variação para a repetibilidade e precisão intermediária do método foram menores que $5 \%$, considera-se o método desenvolvido preciso para ambos os níveis avaliados. ${ }^{1} \mathrm{Da}$ mesma forma, considera-se o método exato e, como a concentração de princípio ativo encontrada no medicamento 3 está cerca de $21,26 \%$ acima do valor indicado pelo fabricante $\left(150.000 \mu \mathrm{g} \mathrm{mL}^{-1}\right)$, sua concentração real é de $181.890 \mu \mathrm{g} \mathrm{mL}^{-1}$.

A robustez do método não foi novamente avaliada para amoxicilina, pois as variações estudadas na robustez da ampicilina visaram estimar a contribuição de mudanças sutis no método analítico e que indicassem possíveis etapas de controle crítico na execução do método. Com os resultados obtidos, já foi possível concluir sobre os potenciais parâmetros críticos a serem controlados a fim de se obter resultados precisos, já que as condições de análise para este fármaco são praticamente as mesmas usadas para a ampicilina.

\section{CONCLUSÕES}

O método cromatográfico desenvolvido e validado para a análise quantitativa de ampicilina e amoxicilina em medicamentos veterinários mostrou-se seletivo, preciso, exato e robusto, de acordo com a legislação vigente no Brasil. Além disso, é um método facilmente aplicável em demais laboratórios de pesquisa e/ou industriais, por usar uma técnica de análise amplamente disseminada, ser rápido, simples, eficiente e com custo moderadamente baixo.

O comércio de medicamentos veterinários no Brasil tem crescido nos últimos anos, porém a fiscalização de tais medicamentos ainda precisa ser mais rigorosa. $\mathrm{O}$ trabalho aqui apresentado mostra que os 3 medicamentos avaliados apresentam concentração do fármaco diferente do indicado na bula, variando de $-12 \%$ a $+21 \%$. Como a administração de um medicamento com quantidade de fármaco divergente da prescrita pode não levar ao efeito desejado (no caso da concentração de fármaco abaixo do valor nominal) ou levar a efeitos tóxicos/residuais (quando é verificada concentração de fármaco mais elevada que indicada na bula), esse problema torna-se ainda mais preocupante no caso de antibióticos, pois são fármacos que, quando administrados de forma errada, podem gerar problemas de desenvolvimento de resistência bacteriana e, caso sejam aplicados em animais destinados a alimentação humana, pode levar à ingestão de resíduos do mesmo pelo consumidor, o que demonstra a importância do método aqui desenvolvido para verificação da quantidade real de fármaco nos medicamentos veterinários.

\section{AGRADECIMENTOS}

À Fundação de Amparo à Pesquisa do Estado de São Paulo (FAPESP) pela concessão da bolsa à G.C. Miguel e pelo apoio financeiro para desenvolvimento do projeto, à CAPES e ao CNPq pelo apoio financeiro para desenvolvimento do projeto.

\section{REFERÊNCIAS}

1. Agência Nacional de Vigilância Sanitária - ANVISA. Programa Nacional de Análise de Resíduos de Medicamentos Veterinários em Alimentos Expostos ao Consumo, Brasília, 2003.

2. Samanidou, V. F.; Evaggelopoulou, E. N.; Papadoyannis, I. N.; J. Sep. Sci. 2006, 29, 1879.

3. http://www.sindan.org.br/sd/sindan/index.html, acessada em Setembro 2012.

4. http://www.abiec.com.br/, acessada em Dezembro 2011.

5. Filch, R. G.; Norrby, S. R.; Greenwood, D.; Antibiotic and Chemotherapy: anti-infective agents and their use in therapy, Churchill Livingston: Edinburgh, 1997.

6. Santos, S. M.; Henriques, M.; Duarte, A. C.; Esteves, V. I. ; Talanta 2007, 71, 731 .

7. Puig, P.; Borrull, F.; Aguillar, C.; Calull, M.; J. Chromatogr. B 2006, 831, 196.

8. Bailón-Pérez, M. I.; García-Campaña, A. M.; Cruces-Blanco, C.; Iruela, M. O.; Electrophoresis 2007, 28, 4082.

9. Bailón-Pérez, M. I.; García-Campaña, A. M.; Cruces-Blanco, C. ; Iruela, M. O.; J. Chromatogr. A 2008 1185, 273.

10. Bailón-Pérez, M. I.; García-Campaña, A. M.; Iruela, M. O.; CrucesBlanco, C. Garcia, L. G.; Electrophoresis 2009, 30, 1708.

11. Nozal, L.; Arce, L.; Ríos, A.; Valcárcel, M.; Anal. Chim. Acta 2004, 523, 21.

12. Belal, F.; El-Kerdawy, M. M.; El-Ashry, S. M.; El-Wasseef, D. R.; Il Farmaco 2000, 55, 680.

13. Amin, S. A.; Il Farmaco 2001, 56, 211.

14. Krasnikoval A. V.; Iozep, A. A.; Pharm. Chem. J. 2003, 37, 49.

15. Benito-Peña, E.; Partal-Rodera, A. I.; León-González, M. E.; MorenoBondi, M.C.; Anal. Chim. Acta 2006, 556, 415.

16. Samanidou, V. F.; Giannakis, D. E.; Papadaki, A.; J. Sep. Sci. 2009, 32, 1302 .

17. Srisom, P.; liawruangrath, B.; Liawruangrath, S.; Chromatographia 2007, 11, 687.

18. Bailón-Pérez, M. I.; García-Campaña, A. M.; Olmo-Iruela, M. Del; Gámiz-Gracia, L.; Cruces-Blanco, C.; J. Chromatogr. A 2009, 1216, 8355.

19. Fernandez-Torres, R.; Consentino, M. O.; Lopez, M. A. B.; Mochon, M. C.; Talanta 2010, 81, 871.

20. Hsieh, S. H.; Huang, H. Y.; Lee, S.; J. Chromatogr. A 2009, 1216, 7186.

21. Smith, S.; Gieseker, C.; Reimschuessel, R.; Decker, C. S.; Carson, M. C.; J. Chromatogr. A 2009, 1216, 8224.

22. Fagerquist, C. K.; Lightfield, A. R.; Rapid Commun. Mass Spectrom. 2003, 17, 660 . 
23. Sorensen, L. K.; Snor, L. K.; Chromatographia, 2001, 53, 367.

24. Abreu, L. R. P.; Ortiz, R. S. M.; de Castro, S. C.; Júnior, J. P.; J. Pharm. Pharm. Sci. 2003, 6, 223.

25. Nelis, H. J.; Vandenbranden,J .; De Kruif, A.; De Leenheerl, A. P.; Antimicrob. Agents Chemother. 1992, 36, 1859.

26. Nelis, H. J.; Vandenbranden, J.; Verhaeghe, B.; De Kruifmm A.; Mattheeuws, D.; De Leenheerl, A. P.; Antimicrob. Agents Chemother. 1992, 36, 1606.

27. Tsou, T. L.; Huang, Y. C.; Lee, C. W.; Lee, A. R.; Wang, H. J.; Chen, S. H.; J. Sep. Sci. 2007, 30, 2407.

28. Sorensen, L. K.; Rasmussen, B. M.; Boison, J. O.; Keng, L.; J. Chromatogr. B 1997, 694, 383.

29. Cha, J. M.; Yang, S.; Carlson, K. H.; J. Chromatogr. A 2006, 1115, 46.

30. Straub, R.; Llnder, M.; Voyksner, R. D.; Anal. Chem. 1994, 66, 3651.

31. Di Corcia, A.; Nazzari, M.; J. Chromatogr. A 2002, 974, 53.

32. Msagati, T. A. M.; Nindi, M. M.; Food Chem. 2007, 100, 836.

33. Holstege, D. M.; Puschner, B.; Whitehead, G.; Galey F. D.; J. Agric. Food Chem. 2002, 50, 406.

34. Riediker, S.; Stadler, R. H.; Anal. Chem. 2001, 73, 1614.

35. Luo, W.; Ang. C. Y. M.; Júnior, H. C. T.; J. Chromatogr. B 1997, 694, 401.

36. Luo, W.; Júnior, E. B. H.; Ang, C. Y. W.; Deck, J.; Freeman, J. P.; Júnior, H. C. T.; J. Agric. Food Chem. 1997, 45, 1264.
37. Pajchel, G.; Pawłowski, K.; Tyski, S.; J. Pharm. Biomed. Anal. 2002, 29, 75.

38. Kirchmann, E.; Welch, L. E.; J. Chromatogr. 1993, 633, 111.

39. Cione, A. P. P.; Liberale, M. J.; Silva, P. M.; Quim. Nova 2010, 33, 203

40. Ma, L.; Kang, W.; Xu, X.; Niu, L.; Shi, H.; Li, S.; J. Anal. Chem. 2012, 67, 219.

41. Freitas, S. K. B.; da Silva, V. L.; Araújo, A. N.; Montenegro, M. C. B. S. M.; Reis, B. F.; Paim, A. P. S.; J. Braz. Chem. Soc. 2011, 22, 279.

42. Ghoulipour, V.; Shokri, M.; Waqif-husain, S.; Acta Chromatogr. 2011, $23,483$.

43. Ribani, M.; Bottoli, C. B. G.; Collins, C. H.; Jardim, I. C. S. F.; Quim. Nova 2004, 27, 771.

44. Cassiano, N. M.; Barreiro, J. C.; Martins, L. R. R.; Oliveira, R. V.; Cass, Q. B.; Quim. Nova 2009, 32, 1021.

45. Olivares, I. R. B.; Lopes, F. A.; TRAC 2012, 35, 109.

46. Núbia, K. P.; Marcelo, M. S.; Quim. Nova 2007, 30, 1359.

47. Dejaegher, B., Heyden, Y. V.; J. Chromatogr. A 2007, 1158, 138.

48. British Pharmacopoeia, ed. 52, 2007.

49. Instituto Nacional de Metrologia, Normalização e Qualidade Industrial (INMETRO); Orientações sobre Validação de Métodos de Ensaios Químicos, DOQ-CGCRE-008, revisão 04, 2011.

50. US Food and Drug Administration (FDA), Department of Health and Human Services, Validation of Chromatographic methods, CMC3, 1994. 